\title{
Comparative study on wavelet filter and thresholding selection for GPS/INS data fusion
}

\begin{abstract}
Navigation and guidance of an autonomous vehicle require determination of the position and velocity of the vehicle. Therefore, fusing the Inertial Navigation System (INS) and Global Positioning System (GPS) is important. Various methods have been applied to smooth and predict the INS and GPS errors. Recently, wavelet de-noising methodologies have been applied to improve the accuracy and reliability of the GPS/INS system. In this work, analysis of real data to identify the optimal wavelet filter for each GPS and INS component for high quality error estimation is presented. A comprehensive comparison of various wavelet thresholding selections with different level of decomposition is conducted to study the effect on GPS/INS error estimation while maintaining the original features of the signal. Results show that while some wavelet filters and thresholding selection algorithms perform better than others on each of the GPS and INS components, no specific parameter selection perform uniformly better than others.
\end{abstract}

Keyword: Vehicular navigation; Inertial navigation; GPS; Wavelet multiresolution analysis; Level of decomposition (LOD); Thresholding 